\title{
PRAXIS HUMANA Y PRAGMÁTICA TRASCENDENTAL DEL LENGUAJE
}

\section{Primeras determinaciones acerca de la lingüisticidad de los actos sobre el trasfondo de la crítica trascendental del sentido.}

\section{Leandro Paolicchi*}

Resumen: La pragmática trascendental del lenguaje de Karl-Otto Apel ha sido principalmente una reconstrucción ( $\mathrm{y}$ fundamentación) de los presupuestos irrebasables del discurso argumentativo. Ella no se ha extendido sobre las acciones en principio no discursivas de los hombres. En el presente trabajo se expone cómo debería entenderse a este tipo de acciones en el marco de dicha pragmática. Así, se presentan aquí las implicancias que tiene para la constitución y explicación de las acciones el ser comprendidas bajo un aspecto central de la pragmática de Apel como es la crítica trascendental del sentido. Con este objetivo, se desarrollan (I) las características generales y esquemáticas que presenta el lenguaje en su rol de "dador" de sentido en la obra de Apel. Luego (II), el artículo se extiende sobre este rol aplicado ahora a las acciones en general de los individuos. Finalmente, y mediante una reflexión sobre la validez implicada en toda articulación lingüística (III), se extraen algunas conclusiones sobre cómo debería entenderse a las acciones en el marco de la pragmática trascendental del lenguaje (IV).

Palabras clave: acción, pragmática, lenguaje, sentido, validez.

Abstract: Karl-Otto Apel's transcendental pragmatics of language has been mainly a reconstruction (and justification) of the inevitable assumptions of argumentative discourse. This pragmatic has said barely anything about non

* Doctor en Filosofía (UNLa). Se desempeña como docente en la Facultad de Humanidades y en la Facultad de Ciencias Económicas y Sociales de la Universidad Nacional de Mar del Plata. En la actualidad es becario posdoctoral de CONICET y se encuentra realizando estudios de posgrado en la Goethe Universität de Frankfurt am Main. Dirección electrónica: leandropaolicchi@yahoo.com 
argumentative action of men. This paper shows what implies for the constitution and explanation of non-argumentative actions of men to be understood under a central aspect of that pragmatic as the transcendental critic of sense (Sinn). To this end, it develops (I) the general and schematic characteristics that language presents in its role of meaning "giver" in the work of Apel. Then (II), the article spreads over this role now applied to the actions of individuals in general. Finally, through a reflection on the validity involved in all linguistic articulation (III), draws some conclusions about how the actions should be understood within the framework of transcendental pragmatics of language (IV).

Key words: action, pragmatics, language, sense, validity.

\section{Lenguaje y mundo en la obra de K.-O. Apel}

En el contexto de las discusiones con el pensamiento de Heidegger y de Wittgenstein en torno a la constitución del sentido, K.-O. Apel ha intentado no sólo la esquematización de algo así como una "antropología del conocimiento", donde se destaca la necesidad de un $a$ priori corporal que es condición de posibilidad para dicha constitución. También es de gran relevancia el hecho de que en cada constitución del sentido se presupone para Apel claramente lo dicho a priori.

El punto de partida para una validez intersubjetiva en la constitución de cualquier sentido radica allí donde la subjetividad de los hombres está constituida simbólicamente. El que alguien pueda atribuir un sentido, aun sin expresarlo, se debe a la existencia de un lenguaje que le precede, que está conformado por una comunidad histórica concreta y nos permite identificar y atribuir a sus "flujos de conciencia" y también a sus acciones una significación determinada. Ahora, si bien esta constitución simbólica es un punto de partida necesario para responder completamente a la pregunta por la valider, no es todavía suficiente. Sin embargo, en la medida en que el lenguaje está allí como condición de posibilidad de apertura de

${ }^{1}$ Cfr. Apel, K.-O., Transformation der Philosophie, Suhrkamp, Frankfurt a. M., 1973, II Bd., p. 96. 
un sentido, están también allí las condiciones contrafácticas en el marco de las cuales se resuelve la validez intersubjetiva de la constitución del sentido y de todo conocimiento verdadero, entendida esta validez en el marco de la pragmática trascendental del lenguaje de Apel como la capacidad de consenso de todos los posibles participantes en el discurso. Esto se verá más adelante.

Pero no sólo en lo referido a la constitución de un sentido subjetivo, es decir, interior a la conciencia, es necesario presuponer una mediación simbólica de los signos lingüísticos. También, y sobre todo, en la constitución de un sentido objetivo de las acciones que se desarrollan en el mundo de la vida es necesario presuponer la mediación simbólica de los signos del lenguaje. Este ha sido un rasgo notable que logró sacar a la luz la filosofía del último Wittgenstein.

En verdad debe concederse, como lo hace el propio $\mathrm{Apel}^{2}$, que la reflexión sobre la constitución lingüística del mundo, y con ello de la implicación misma del hombre en la constitución de éste, es algo que permanece fuera de la actitud natural o espontánea del hombre en su vida cotidiana. Cuando nos desenvolvemos en nuestra vida diaria, no reflexionamos a menudo sobre el lenguaje y la relación "creadora" que éste puede llegar a tener con determinados hechos del mundo. La actitud contraria, es decir, la tendencia a ver el mundo en su materialidad intacta fuera de la conciencia sin intervención del lenguaje, viene determinada en parte por el uso mismo del lenguaje. Es decir, el lenguaje nos “distancia” del mundo y de las cosas, y tiende a hacernos creer que los fenómenos del mundo se constituyen sin la intervención de éste. Asimismo, esta forma de percibir el mundo aparece consagrada en ciertas concepciones filosóficas sobre el lenguaje como las que predominaron en la Antigüedad, la Edad Media $^{3}$ y sobre todo en la tradición del atomismo lógico, de manera paradigmática en el Wittgenstein del Tractatus Logico-Philosophicus.

Con el objetivo de ilustrar la intervención del lenguaje en la constitución del sentido, Apel trae a colación el ejemplo de la cópula "es",

\footnotetext{
${ }^{2}$ Cfr. Apel, K.-O., Transformation der Philosophie, I, ed. cit., p. 243 y ss.

${ }^{3}$ Ibid., loc. cit.
} 
cuya función es abordada en la discusión con el Wittgenstein del Tractatus. Para el Wittgenstein de este período, algunos enunciados con la cópula "es" son considerados una simple tautología y no la operación sintética de una conciencia. Para Apel, la existencia de la cópula "es", por ejemplo en la oración "el libro está sobre la mesa", indica precisamente que ese estado de cosas no se constituye con independencia de la cópula "es" ni con independencia de la implicación de un hombre (y su lenguaje) concreto. Cito a Apel:

A la lógica le parece como si, por ejemplo, el estado de cosas: "el libro está (es) sobre la mesa" se diera de una forma acabada y totalmente independiente del "es" de la cópula y, con ello, del "ser en el mundo" del hombre. El "es", como ya se indicó, parece expresar en todos los casos, en cuanto reconocimiento de estados de cosas qua hechos independientes del hombre, una relación con el hombre ${ }^{4}$.

Este ejemplo se le aparece a Apel como la prueba cabal de la implicación del hombre (y de su lenguaje) en la constitución y significatividad de los objetos como tales. Es decir, no sólo mienta la cópula "es" una relación de las cosas del mundo con el hombre, sino que ella fundamentalmente demuestra la condición de constitución que el hombre (y su lenguaje) establece con el mundo para que haya estados de cosas dentro de él.

Este planteo no ha sido ideado por Apel, sino que lo toma -como él mismo lo explica- de su maestro Erich Rothacker, y de alguna manera ha sido llevado a su máxima complejidad conceptual por Martin Heidegger. De hecho, puede decirse que Apel toma en gran parte el desarrollo en torno a la cuestión de la constitución del sentido del programa heideggeriano, continuado luego en la hermenéutica de Gadamer. De alguna manera, los planteos heideggerianos le parecen a

4 Apel, K.-O., Transformation der Philosopbie, I, ed. cit., p. 244. La traducción de todos los fragmentos citados de la obra de Apel me corresponde. 
Apel la forma más acabada de explicar la constitución histórica del sentido y por ello los adopta casi en su totalidad.

Sin embargo, los análisis heideggerianos que se proponen explícitamente como una continuación de los planteos trascendentales kantianos ${ }^{5}$ adolecen de una falta grave. De hecho, para Apel, Heidegger entiende sólo una parte de los planteos kantianos en Kritik der reinen Vernunft, soslayando al mismo tiempo otro aspecto que Apel considera determinante y que es el que él mismo se propone sacar a la luz contra los planteos historicistas como los de Heidegger y los de Gadamer. De algún modo, los intentos de Heidegger omiten o, mejor aún, intentan fundir dos aspectos clave que estaban presentes en la obra de Kant. Por un lado, el problema de la constitución o génesis del sentido, pero, por otro, la cuestión fundamental de la validez intersubjetiva de todo conocimiento de objetos ${ }^{6}$, o como Apel explícitamente lo plantea: "la diferencia entre la pregunta por la constitución del sentido lingüísticamente mediado y la pregunta por la posible justificación de la validez de nuestro conocimiento"?.

Es posible detectar un pensamiento similar en Habermas, quien se alinea claramente detrás de las adquisiciones del giro lingüístico para criticar una concepción del lenguaje heredada de la filosofía analítica y del positivismo lógico. En este sentido, Habermas retoma una concepción del lenguaje heredada de Heidegger, y en muchos puntos común con la de Gadamer, para resaltar la dimensión intersubjetiva del lenguaje y en consonancia con ello la capacidad del lenguaje de "abrir un mundo"8. Pero

${ }^{5}$ Esto vale especialmente para las obras tempranas de Heidegger como Kant und das Problem der Metaphysik y Sein und Zeit. Luego de la "Kehre" es más difícil rastrear esta continuidad. Sin embargo, Lafont sostiene que Heidegger sigue apegado todavía a la distinción a priori - a posteriori. Véase Sprache und Welterschließung. Zur linguistischen Wende der Hermeneutik Heideggers, Suhrkamp, Frankfurt am Main, 1994.

${ }^{6}$ Cfr. Apel, K.-O., Auseinandersetzungen in Erprobung des transzendentalpragmatischen Ansatzes, Suhrkamp, Frankfurt am Main, 1998, p. 511 y 537 passim.

${ }^{7}$ Ibid., p. 548.

${ }^{8}$ Cfr. Lafont, C., La Razón como Lenguaje. Una revisión del "giro lingüístico" en la filosofia del lenguaje alemana, Madrid, Visor, 1993. 
de alguna manera, Habermas, si bien rescata y hace suyos los aportes que en términos de constitución del sentido ha hecho la hermenéutica de Humboldt a Gadamer, quiere asimismo evitar otro conjunto de características que traen consigo estas filosofías y que eliminan precisamente las dimensiones sobre las cuales quiere hacer hincapié su propia propuesta.

Así, Habermas buscará combatir la historicidad y relatividad que traen consigo las propuestas de Heidegger y Gadamer rescatando una dimensión de validez, y por lo tanto de crítica y de universalidad, inherente al lenguaje mismo. Me parece que estas dos características definen en esencia a los planteos tanto de Habermas como de Apel. Es decir, por un lado la necesidad de adoptar los aportes indispensables que tanto en cuestiones de génesis como de constitución histórica del sentido ha hecho una tradición que se remonta a Humboldt y pasa por Heidegger hasta llegar a Gadamer. Pero por otro lado, la necesidad también imprescindible de evitar otro conjunto de particularidades que trae consigo esta forma de concebir al lenguaje únicamente como apertura del mundo. Para estas concepciones, como las de Heidegger o Gadamer, el lenguaje no sólo es portador de una forma de interpretar el mundo, de una manera de determinarse los entes dentro de él, de una determinada "precomprensión del ser" como la llama Heidegger, sino de una historicidad que somete toda pregunta por la validez universal a una relatividad bistórica insuperable.

Pero, retomando a Apel y su proyecto de una antropología del conocimiento, que en verdad luego de los escritos iniciales en Transformation der Philosopbie no se ha vuelto a tratar ${ }^{9}$, Apel desarrolla la idea central a la hermenéutica de la constitución lingüística del mundo y a partir de allí la constitución intersubjetiva de todo sentido. Así dice con respecto al primer punto:

9 Puede verse quizás un indicio de ese proyecto en K.-O. Apel, "OrtoderOrtlosigkeit der Vernunft? Leibapriori versus Reflexionsapriori der Erkenntnis", en W-J. Cramm / G. Keil (Hrsg.), Der Ort der Vernunft in einer natürlichen Welt,Velbrück Wissenschaft, Weilerswist, 2008. 
Tenemos que comprender los datos del mundo mismos, en cuyo contexto aparece la conducta que queremos comprender, a partir de la comprensión intencional. El mundo ya no es la "existencia de las cosas, en cuanto constituyen una conexión según leyes (en el sentido de la ciencia natural)" (Kant), sino la "situación global" de un determinado "ser-en-el-mundo" (Heidegger), en la que podamos participar mediante la comprensión lingüistica. ${ }^{10}$

Es decir, para Apel podemos comprender la conducta desde una dimensión intencional e implicándonos en una determinada "situación global", que no es producto sino de nuestra situación de "ser-en-elmundo", porque existe una constitución común a priori de nuestra subjetividad y del mundo que es el lenguaje. El lenguaje constituye para la pragmática trascendental una condición de posibilidad de la experiencia, de los objetos de la experiencia, del conocimiento y de la objetividad (sentido) de esos objetos. Se ve aquí replicada la estructura gnoseológica kantiana, reemplazando ahora el a priori de la subjetividad de la conciencia por el a priori lingüístico de una comunidad de comunicación. Es importante tener en cuenta este paralelismo, pues a Kant se remite Apel cuando critica aquello que de alguna manera viene anexado a una apertura lingüística del mundo, es decir, cuando critica en Heidegger y Gadamer la omisión de la pregunta por la validez de la objetividad del conocimiento y su asimilación a la pregunta por la constitución histórica del sentido en el desocultamiento del ser ${ }^{11}$.

Podríamos decir entonces que desde el punto de vista de las perspectivas de Apel y Habermas el lenguaje participa en el grado cero del conocimiento, en la constitución misma de los datos. Apel se encarga de dejarlo bien en claro en varias oportunidades y sobre todo en aquellos pasajes en donde discutiendo con las epistemologías positivistas y en el marco de la discusión sobre el alcance de las ciencias hermenéuticas

10 Apel, K.-O., Transformation der Philosophie, II, ed. cit, p. 108. Las cursivas me pertenecen.

${ }^{11}$ Apel, K.-O., Auseinandersetzungen in Erprobung des transzendentalpragmatischen Ansatzes, ed. cit., pp. 505-568 y 569-608. 
destaca el lugar del lenguaje, bajo la forma de una comunidad de comunicación, como presupuesto de todo conocimiento en la dimensión sujeto-objeto y como "metadimensión de la descripción de los datos del mundo"12. Así, por ejemplo, cuando en el marco de los estudios sobre Peirce se refiere a la comunidad (de lenguaje) como una condición de posibilidad de una definición de la realidad ${ }^{13}$ o en el marco de las discusiones con Wittgenstein se describe al lenguaje como condición de posibilidad de algo en cuanto algo ${ }^{14}$.

\section{El lenguaje y el sentido de las acciones}

Ahora bien, si se debe presuponer necesariamente una comunidad (real e ideal) de lenguaje en toda concepción y sentido de lo real, esta presuposición es también naturalmente válida para la identificación del sentido de las acciones que transcurren como parte de la realidad $^{15}$. Es decir, si tengo que presuponer una comunidad de comunicación para una atribución de sentido a todos los objetos de la realidad, la tengo que presuponer naturalmente para la atribución de un sentido a las acciones de los hombres que forman parte de esa realidad. No puedo otorgarle un sentido tampoco a una acción sin presuponer una comunidad intersubjetivamente estructurada a través del lenguaje. Esto había sido bien detectado por Wittgenstein, aunque no de una manera trascendental.

En este sentido es importante el hecho de que no sólo el punto de vista del observador, sino también los actores mismos que llevan adelante sus acciones en el mundo de la vida deben presuponer necesariamente una comunidad con la cual comparten un lenguaje común. No les es

\footnotetext{
${ }_{12}$ Apel, K.-O., Transformation der Pbilosophie, II, ed. cit., p. 114.

13 Cfr. Apel, K.-O., El camino del pensamiento de Charles S. Peirce, Madrid, Visor, 1997, pp. $52-53$.

${ }_{14}$ Apel, K.-O., Transformation der Philosopbie, I, p. 328.

${ }^{15}$ Apel, K.-O., Semiótica Filosófica, ed. cit., p. 297.
} 
posible a los actores atribuir un sentido a sus acciones, es decir, saber propiamente que actúan, sin presuponer al mismo tiempo una comunidad de comunicación. Cuando un actor traza un plan para su acción, busca saber lo que ya hizo o lo que está haciendo en ese momento, es decir, intenta darle un sentido a sus acciones, debe contar con que ello es posible por una comunidad real de lenguaje que le facilita los medios (lingüísticos) para dicha identificación. Si no existiera una comunidad estructurada intersubjetivamente a través del lenguaje el actor no dispondría de los medios para identificar sus propias acciones, para otorgarle un sentido a lo que hace. Por ejemplo, un sujeto dice luego de cruzar unas vías con su auto: "fui completamente imprudente al cruzar las vías con la barrera baja". Ahora bien, dicho individuo pudo darle un sentido a su acción, es decir, pudo identificar su acción, pues disponía de los medios lingüísticos de una comunidad de comunicación en la cual él fue socializado. Si no conociera el significado de la palabra "prudente" y su uso en su comunidad, así como el uso de las barreras bajas no podría haberle dado a su acción ningún tipo de sentido. Sin la disposición de ese lenguaje, dicho sujeto no hubiera podido reconocer su acción, no hubiera sabido lo que hizo. Con esto se arriba a uno de los pensamientos centrales de la crítica trascendental del sentido. Cito a Apel: "La capacidad misma de pensar depende a priori de la "forma interna" o la "gramática profunda" del lenguaje, cuyo uso está "entretejido" (Wittgenstein) con las reglas del obrar con sentido y con las reglas de la comprensión del mundo, incluida la autocomprensión."16

${ }^{16}$ Apel, K.-O., Transformation der Philosophie, II, p. 315. Véase también Ibíd., pp. 393, 398 y 400; Apel, K.-O., "Faktische Anerkennung oder einsehbar notwendige Anerkennung? Beruht der Ansatz der transzendentalpragmatischen Diskursethik auf einem intellektualistischen Fehlschluß?" en Apel, Karl-Otto (Hg.) in Verbindung mit Riccardo Pozzo, Zur Rekonstruktion der praktischen Pbilosophie. Gedenkschrift für Karl-Heinz Ilting, Stuttgart-Bad Cannstatt, Frommann-Holzboog, 1990, p. 100; Id., Auseinandersetzungen in Erprobung des transzendentalpragmatischen Ansatzes, ed. cit. y Øfsti, A., "Das Sprachspiel-Idiom und die Einheit der Vernunft. Bemerkungen zu Apels Wittgensteinkritik", en Dorschel, Andreas: 
Todo pensar y obrar con sentido presupone necesariamente una comunidad real (y una ideal) de comunicación. Es imposible para un actor pensar que está realizando una acción determinada, pero la lleva a cabo en la más absoluta soledad de la conciencia y sin la existencia de otros sujetos con los que comparte un lenguaje. Si esto último fuera verdad, el actor no dispondría de los medios para reflexionar sobre su propia acción ni atribuirle un sentido, es decir, no sabría lo que hace. Cuando un actor tiene la intención de realizar una acción, es decir, le atribuye un sentido a ella, debe contar en esa intencionalidad irremediablemente con la presencia de un lenguaje que le ha sido otorgado en función de su socialización en una comunidad lingüística real. Cuando alguien, por ejemplo, expresa su intención (aunque sea internamente) de realizar una llamada telefónica y dice "voy a llamar por teléfono" puede hacerlo obviamente pues dispone de un lenguaje "adquirido" en una comunidad de lenguaje. Pero no existe otra forma de dar un sentido que no sea a través de los signos lingüísticos. Cuando se expresa la intención de hacer algo y se le da un sentido a las acciones que se realizan debe presuponerse necesariamente la presencia del lenguaje. Esto lo confirma Apel cuando señala que: "Tampoco los signos lingüísticos se cuentan entre los objetos del conocimiento, porque también ellos están ya presupuestos, como condición de posibilidad de cualquier intención con sentido."17

Ahora bien, esta circunstancia puesta al descubierto por la crítica trascendental del sentido conduce al otro punto importante que debe mencionarse aquí. Así como un lenguaje puede ser identificado como tal sólo en el marco de una determinada comunidad de lenguaje, en el caso de una acción intencional sucede exactamente lo mismo. Tal como lo explicita la crítica trascendental del sentido, una acción propiamente bumana sólo puede ser identificada y llevada a cabo conscientemente en el contexto de una comunidad de lenguaje. Actuar con sentido sólo puede ser entendido como tal teniendo como referencia ciertas reglas, normas o prácticas previas por las cuales ese actuar se guía, ya sea haciendo estrictamente lo que ellas

Traszendentalpragmatik, Frankfurt am Main, Suhrkamp, 1993, p. 63 y 72. Las cursivas me pertenecen.

${ }^{17}$ Apel, K.-O., Transformation der Philosophie, II, p. 99. Las cursivas me pertenecen. 
determinan, modificando parcialmente su sentido o apartándose completamente de ellas. Cuando alguien, en ciertas sociedades, eleva su mano a la altura de su hombro frente a un auto de color negro, amarillo o ambos y se sube por la puerta de atrás, sabemos que esa persona está pagando un servicio en un auto que no le pertenece para trasladarse a alguna parte, es decir, está tomando un taxi. Ahora bien, sabemos que ése es el sentido de tal acción porque conocemos las reglas mediante las cuales en ciertas sociedades se toma un taxi: el auto apto para este servicio debe estar identificado de determinada manera, debe estar vacío, debo hacerme ver con la mano extendida frente a él, etc. Si no supiéramos las reglas mediante las cuales se toma un taxi o si ni siquiera supiéramos de la existencia de taxis no podríamos comprender el sentido de esa conducta. Sería para nosotros tan sólo un conjunto de movimientos corporales sin sentido o con un sentido que desconocemos.

Bajo este punto de vista, podríamos decir que no bay diferencia entre una conducta humana y un lenguaje. Ambos sólo pueden ser entendidos como tales y llevados a cabo sólo en función de pautas, reglas o normas específicas determinadas por una comunidad de lenguaje. Este hecho es el que permite diferenciar las acciones humanas (lingüísticas o no) de los acontecimientos naturales ${ }^{18}$, tales como la caída de un árbol o el aumento del caudal de un río. Las primeras aparecen como tales sólo en referencia a las reglas y a las normas de conducta de una comunidad de lenguaje. Es por esto también que le cabe al lenguaje en la pragmática trascendental el papel de a priori trascendental de los fenómenos del mundo de la vida. Es decir, el lenguaje es un a priori que nos permite "tener" acciones humanas con sentido y nos permite diferenciarlas del resto de los acontecimientos naturales.

Ahora bien, el hecho de que toda acción con sentido sólo sea posible por la existencia de un lenguaje posibilita entender a las acciones como lenguaje. Naturalmente, esta última afirmación no significa que las acciones sean estrictamente lenguaje. No se propone aquí una simple

18 Cfr. Kuhlmann, W., Reflexive Letz̧tbegrünndung, Untersuchungen zur Transzendentalpragmatik, Frankfurt am Main, Suhrkamp, pp. 156, 157 y 161. 
identificación de dos ámbitos que son evidentemente distintos. Aquí sólo se busca presentar la idea de que tanto las acciones como el lenguaje comparten estructuras similares que permiten entenderlos como lo que son, es decir, acciones humanas y lenguaje. Es la constitución lingüística del mundo de las acciones humanas lo que conduce a que no podamos entenderlas sino con las mismas condiciones de posibilidad que un lenguaje. Esto ha sido claramente afirmado por Apel: "La conducta misma tiene el carácter de un lenguaje y por lo tanto tiene que ser comprendida."19 Es decir, si no entendemos a las acciones con sentido con una estructura similar al lenguaje no podemos concebir siquiera la idea de una acción propiamente bumana. Debemos resignarnos a sumergir las acciones con sentido en el marco más amplio de los "acontecimientos naturales".

Sin embargo, si no se quiere recaer en esto último, debe concebirse al lado de la noción central a la modernidad de experiencia objetiva, el concepto de experiencia comunicativa, como el nuevo marco donde es posible concebir y comprender la praxis del hombre ${ }^{20}$. El mundo de las acciones y la experiencia de las acciones no son una experiencia objetiva de una conciencia subjetiva, sino una experiencia comunicativa ${ }^{21}$. Sólo en el marco de este tipo de experiencia podemos concebir acciones con sentido y por lo tanto verlas como un lenguaje. Podemos percibir (y describir) acciones, pues siguen la misma lógica de quien las describe en tanto sujeto de habla ${ }^{22}$ y por ello adoptan específicamente esta forma. Las acciones leídas bajo el concepto de experiencia comunicativa tienen el carácter de un lenguaje.

De todas formas, debe quedar aquí en claro algo muy importante. Más allá de que las acciones en el mundo de la vida deban ser entendidas

19 Apel, K.-O., Transformation der Philosophie, II, ed. cit., p. 46. Las cursivas me pertenecen.

${ }^{20}$ Véase Habermas, J., "Was heisst Universalpragmatik?”, en Apel, Karl-Otto et al., Hermeneutik und Idiologiekritik, Frankfurt am Main, Suhrkamp, 1971, pp. 183 188; Böhler, D., Rekonstruktive Pragmatik, ed. cit., pp. 296 - 302 y Kuhlmann, W., Reflexion und kommunikative Erfabrung, Frankfurt am Main, Suhrkamp, 1975.

${ }^{21}$ Cfr. Böhler, D., Rekonstruktive Pragmatik, ed. cit., p. 296.

22 Ibid., p. 300. 
lingüísticamente $y$, por lo tanto, que sea necesaria una aproximación bermenéutica que nos ponga en condiciones de reconstruir el sentido atribuido por los individuos a sus acciones, este tipo de perspectiva de análisis no es la única posible para examinar la praxis del hombre. Esta cuestión forma parte de las extensas discusiones que Apel ha entablado en la tradición de la controversia entre las ciencias naturales y las ciencias del espíritu y luego las ciencias sociales ${ }^{23}$. No concierne de una manera directa a nuestro trabajo, pero debe quedar en claro que las acciones humanas son también pasibles de una aproximación explicativa ${ }^{24}$, típica de las ciencias naturales. Poner de manifiesto la necesidad de una aproximación hermenéutica no significa negar la posibilidad de un análisis explicativo de la conducta humana. En algún punto, esta última afirmación resulta casi una verdad evidente, como lo demuestra la proliferación de ciencias de las denominadas "humanas" que hacen un uso exitoso y exhaustivo de los métodos explicativos de las ciencias naturales, como en la ciencia política, la economía o la psicología.

También para Habermas el lenguaje es un marco constitutivo de las estructuras del mundo de la vida. En numerosas oportunidades a lo largo de Theorie des kommunikativen Handelns, y sobre todo en aquellos pasajes sobre los que se extiende acerca de la constitución del mundo de la vida, Habermas resalta el papel del lenguaje como marco trascendental que constituye sus estructuras. Esta idea forma parte central del alineamiento habermasiano detrás de la tradición lingüística de Humboldt y Gadamer, tradición que tiene en el centro de su consideración al lenguaje en su capacidad de apertura del mundo ${ }^{25}$.

Dice Habermas en Theorie des kommunikativen Handelns:

${ }^{23}$ Cfr. Apel, K.-O., Die Erklären : Verstehen Kontroverse in transzendentalpragmatischer Sicht, Frankfurt am Main, Suhrkamp, 1979. Véase también Habermas, J., Zur Logik der Sozialwissenschaften, Frankfurt am Main, Suhrkamp, 1982.

${ }^{24}$ Cfr. Krings, H., "Kann man die Natur verstehen?" en Kuhlmann, Wolfgang und Böhler, Dietrich (Hrsg.), Kommunikation und Reflexion. Zur Diskussion der Transzendentalpragmatik Antworten auf Karl-Otto Apel, Frankfurt am Main, Suhrkamp, 1982, pp. 387 - 389.

${ }^{25}$ Cfr. Lafont, C., op. cit., p. 183. 
$\mathrm{Si}$, como es habitual en la tradición que se remonta a Humboldt, suponemos una conexión interna entre las estructuras del mundo de la vida y las estructuras de la imagen lingüística del mundo, al lenguaje y a la tradición cultural les compete en cierto modo un papel trascendental frente a todo aquello que puede convertirse en componente de una situación ${ }^{26}$.

Es decir, nuevamente aquí como en Kant aparece un a priori como condición de posibilidad de los objetos de la experiencia, pero no es un $a$ priori lógico trascendental de la conciencia, como en Kant, sino un a priori lingüístico. En otras palabras, se ve claramente la continuación de la tradición trascendental kantiana, pero ahora ya dentro de la tradición del giro lingüístico de la filosofía del siglo XX. El lenguaje es ahora en los planteos de Apel y Habermas el que funciona como condición de posibilidad de la experiencia y como condición de posibilidad de los objetos de la experiencia. Es decir, la función de "apertura" del mundo atribuida al lenguaje significa que todo fenómeno de la experiencia se me da como tal dentro de los marcos categoriales impuestos a priori por el lenguaje. El lenguaje permite que el hombre tenga un mundo con un conjunto de sucesos que lo constituyen y "abre" un mundo para el hombre. El que algo se me dé como algo en la experiencia sólo es posible porque el lenguaje así lo posibilita. El lenguaje hace posible que haya "estados de cosas" dentro del mundo y que éstos se manifiesten como experiencia ${ }^{27}$.

\section{Lenguaje y Validez}

La clave en el conjunto de estas remisiones es que, tanto en Kant como en Apel y Habermas, las estructuras que funcionan como condición

\footnotetext{
${ }^{26}$ Habermas, J., Theorie des kommunikativen Handelns, ed.cit., Bd. II, p. 190. ${ }_{27}$ Cfr. Apel, K.-O., Auseinandersetzungen in Erprobung des transzendentalpragmatischen Ansatzes, ed. cit., p. 540.
} 
de posibilidad de la experiencia, y de los objetos dentro de ella, son las mismas que aseguran la objetividad del conocimiento de la experiencia. Esta idea es un punto fundamental para evitar precisamente aquello mismo que adviene con la aceptación de los planteos heideggerianos y gadamerianos, a saber, el peligro de una historicidad radical y un relativismo que subsuma toda posible pregunta por una validez universal del conocimiento. Es decir, en el hecho de considerar al lenguaje en su función de "apertura del mundo" se corre el riesgo de asumir la historicidad inherente al lenguaje concreto y transformar su estructura transmitida por la tradición cultural, en términos heideggerianos en un "acontecer de la verdad" o como dice Lafont: "en una instancia normativa, fuente originaria de la validez de todo saber intramundano y, sin embargo, históricamente cambiante (al haber sido descentralizada en forma de tradición cultural)."28 La historicidad cambiante en su desarrollo, vehiculizada a través de los lenguajes concretos y naturales, sería, en palabras de Lafont, el único parámetro normativo con el cual dictaminar la validez o invalidez de todo conocimiento. Pero con ello la validez se alteraría en el curso de la historia dando lugar a un historicismo y a un relativismo radical.

Para Apel, concebir al lenguaje de esta manera significaría que toda constitución del sentido del mundo temporal e histórico, vehiculizada a través del lenguaje, determina los límites de las preguntas posibles y por lo tanto de los juicios verdaderos o falsos ${ }^{29}$. Sin embargo, para Apel, Heidegger sólo se queda en un aspecto del problema kantiano de la pregunta por las condiciones de validez del conocimiento. Kant se pregunta cómo se dan los objetos, pero también cómo se valida intersubjetivamente el conocimiento de esos objetos ${ }^{30}$. Con ello Heidegger se ve atrapado en la aporía referente a la validez de su propia teoría y a las pretensiones que con ella eleva. Con esto se arriba al punto clave en la crítica trascendental del sentido tal como está planteada en la pragmática trascendental de Apel.

28 Lafont, C., op. cit., p. 194.

29 Cfr. Apel, K.-O., Auseinandersetzungen in Erprobung des transzendentalpragmatischen Ansatzes, ed. cit., p. 543.

${ }^{30}$ Ibid., p. 511. 
Desde este punto de vista, ¿cómo deben entenderse los análisis heideggerianos de la constitución histórica del sentido? ¿Cómo deben resolverse las pretensiones de validez que la propia teoría beideggeriana eleva? En este punto, Apel se pregunta si puede volverse la pretensión de verdad de los análisis del a priori de la facticidad de Heidegger al mismo a priori, en el sentido de una preestructura de la comprensión.

La respuesta que debe dar la pragmática trascendental es que, si se quiere evitar una inconsistencia pragmática negando en la dimensión performativa de la enunciación el a priori de la facticidad que se afirma a nivel proposicional, la pretensión de verdad que con los estudios del a priori de la facticidad se eleva debe valer contrafácticamente ${ }^{31}$. Pero inevitablemente con esta reflexión pragmático-trascendental sobre las pretensiones de validez contrafácticas de las investigaciones heideggerianas se pone un límite a aquella relativización histórica de toda verdad que se desprende de la obra de Heidegger. La pretensión de verdad es ahora una condición de posibilidad pragmático-trascendental de todo conocimiento posible ${ }^{32}$ no relativizable a ninguna apertura bistórica del sentido. De todas formas, ello no significa rechazar los planteos heideggerianos como un todo. Al contrario, está en el centro de la transformación pragmático-trascendental de la filosofía rescatar dichos análisis como fundamentales para ilustrar la constitución lingüística del sentido del mundo ${ }^{33}$. Sin olvidar, sin embargo, que ya con el estudio de la constitución lingüística del mundo se han reconocido presupuestos trascendentales necesarios del discurso argumentativo que representan las condiciones ideales contrafácticas de toda posible validez y crítica. La reconstrucción y fundamentación de estos presupuestos forma parte de la tarea tradicional de la pragmática trascendental.

${ }^{31}$ Ibid., p. 554 y 560.

${ }^{32}$ Ibid., p. 560.

${ }^{33}$ Ibid., p. 567. 


\section{Algunas conclusiones}

La crítica trascendental del sentido de la pragmática trascendental del lenguaje aplicada ahora a las acciones no discursivas de los hombres nos ha llevado a las siguientes conclusiones:

1) Para la identificación de un sentido en las acciones humanas, es decir, para identificarlas propiamente como acciones y no como cualquier otro fenómeno natural, debemos presuponer necesariamente la existencia de una comunidad real de comunicación. Sólo en el marco de esta comunidad se me permite otorgarle un sentido a la acción, en una primera instancia desde el punto de vista de un observador-participante.

2) Sin embargo, también en otro nivel fundamental es posible detectar este tipo de identificación. No sólo desde el punto de vista del observador participante, sino también desde la perspectiva del actor mismo puede constatarse esto. En la medida en que el actor no sólo actúa ciegamente, sino que puede comprender lo que hace y puede atribuirle un sentido a sus acciones debe presuponer necesariamente una comunidad lingüística. Sólo en el marco de un lenguaje real y socializado le es posible al actor comprender lo que hace e identificar sus acciones como de un tipo específico, por ejemplo, tomar un té o prender la computadora. Sin la intervención de un lenguaje real esto le sería imposible a un actor. Esta idea puede expresarse también de la siguiente manera: un actor es aquel que puede responder a las preguntas del tipo “¿qué está Ud. haciendo?” o "¿con qué objetivo realiza esa acción?", es decir, actor es aquel que es capaz de expresar lingüísticamente el sentido de sus acciones en principio no lingüísticas. Pero esto puede hacerlo, pues en la medida en que le atribuye un sentido a su acción, es decir, realiza concientemente una acción específica y no otra, presupone ya una comunidad (real e ideal) de comunicación.

3) No obstante, como se acaba de ver en este mismo trabajo, con la constitución lingüística de todo sentido están también dadas las condiciones contrafácticas en el marco de las cuales debe resolverse la pregunta central sobre la validez y la objetividad posible de todo 
conocimiento verdadero, de toda comprensión del sentido de esas acciones. La articulación lingüística de todo sentido remite contrafácticamente a su validez intersubjetiva en el sentido de un consenso de todos los participantes posibles en un discurso argumentativo.

Esta idea, central a la pragmática trascendental, puede ser probada en la discusión de Apel con Heidegger. Sin embargo, desde un punto de vista estrictamente pragmático trascendental, puede y debe ser probada también a través de las mismas acciones que se llevan adelante con este escrito. En la medida en que es una acción humana y por lo tanto se le atribuye un sentido determinado, debe presuponer necesariamente una comunidad real de interlocutores que comparten un lenguaje. Pero en la medida en que no sólo pretende tener sentido, sino que eleva también una pretensión de verdad remite necesaria y contrafácticamente a una comunidad ideal de argumentantes a la cual están dirigidas todas las pretensiones que con este trabajo se elevan y en el marco de la cual se resolverá su validez. Negar con este escrito en su dimensión proposicional la existencia de una comunidad real e ideal de interlocutores llevaría irremediablemente a cometer una inconsistencia pragmática.

4) Por último, la tesis de que las acciones como tales sólo se dan en el marco de una comunidad de lenguaje ha llevado a la idea de que también las acciones tienen el carácter de un lenguaje, es decir, comparten estructuras comunes que bacen posible entenderlos como tales. Este pensamiento está en el centro del concepto desarrollado por la pragmática trascendental (y no sólo ella) de experiencia comunicativa.

Por restricciones de espacio queda para trabajos futuros desentrañar en su totalidad el alcance conceptual que tiene entender la conducta humana como un lenguaje y qué tipo de implicancias normativas es posible extraer de él.

Recibido: 12/2012. Aceptado: 04/2103 